\title{
Análise de tendências nas séries históricas de precipitação e curva de permanência de vazão no município Cachoeira do Piriá, Pará
}

\author{
Analysis of trends in the historical series of precipitation and flow stay curve in Cachoeira do Piriá, \\ Pará
}

Análisis de tendencias en serie histórica de precipitación y curva de estancia de flujo en Cachoeira do Piriá, Pará

Recebido: 06/05/2021 | Revisado: 11/05/2021 | Aceito: 15/05/2021 | Publicado: 04/06/2021

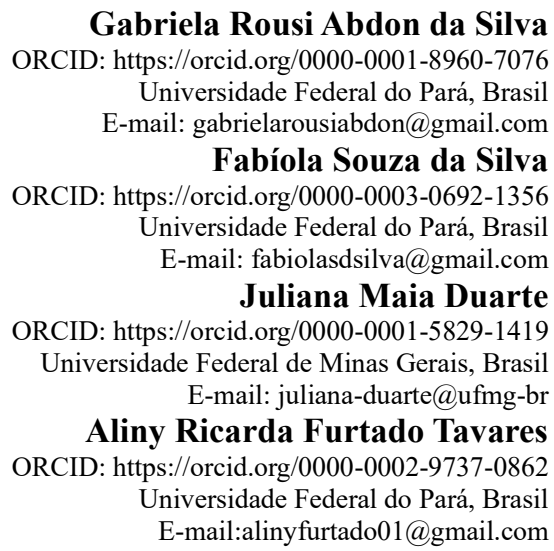

\section{Resumo}

O Norte do Brasil apresenta altos índices pluviométricos, o que está relacionado a presença da floresta Amazônica. Em vista disso é necessário que estudos que analisem o comportamento hidrológico em bacias hidrográficas desta região sejam desenvolvidos, para que estes possam ser utilizados, posteriormente, como ferramentas e bases pra outras tipologias de estudos ou para análise de viabilidades de obras de modo geral. Dois dos principais componentes do ciclo hidrológico são as precipitações e as vazões dos corpos hídricos. Desta forma a presente pesquisa realizou uma análise acerca da estação pluviométrica de código (146008) e fluviométrica de código (32620000), ambas localizadas no munícipio de Cachoeira do Piriá, estado do estado do Pará, com o objetivo de analisar a influência das precipitações nas vazões do rio da bacia hidrográfica através da construção de um hietograma, realizando também a avaliação de normal climatologia e tendência para a série histórica dos dados de chuva, utilizando os métodos Linear, Mann Kendall e Sen's Slope. Além disso, para os dados de vazão foi elaborado a curva de permanência. Por fim, foi observado, a partir dos dados pluviométricos, que a precipitação no município não apresenta tendência e, a partir dos dados fluviométricos, foi possível observar que sua vazão em $95 \%$ dos casos é de, aproximadamente, $200 \mathrm{~m}^{3} / \mathrm{s}$.

Palavras-chave: Precipitação; Tendência; Vazão; Cheias.

\begin{abstract}
Northern Brazil has high rainfall, which is related to the presence of the Amazon rainforest. In view of this, it is necessary that studies that analyze the hydrological behavior in hydrographic basins of this region be developed, since these can be used, later, as tools and bases for other types of studies or for analyzing the viability of works in general. Two of the main components of the hydrological cycle are precipitations and flows from water bodies. In this way, the present research carried out an analysis about the code pluviometric station (146008) and code fluviometric station (32620000), both located in the municipality of Cachoeira do Piriá, state of Pará, with the objective of analyzing the influence of precipitations in the river flows in the hydrographic basin through the construction of a hietogram, also carrying out the assessment of normal climatology and trend for the historical series of rain data, using the Linear, Mann Kendall and Sen's Slope methods. For the flow data, the permanence curve was elaborated. Finally, it was observed, from the pluviometric data, that the precipitation in the municipality does not present a trend and, from the fluviometric data, it was possible to observe that its flow in $95 \%$ of the cases is, approximately, $200 \mathrm{~m}^{3} / \mathrm{s}$.
\end{abstract}

Keywords: Rainfall; Trend; Flow; Floods. 


\begin{abstract}
Resumen
El norte de Brasil tiene altas precipitaciones, lo que está relacionado con la presencia de la selva amazónica. Ante esto, es necesario que se desarrollen estudios que analicen el comportamiento hidrológico en las cuencas hidrográficas de esta región, ya que estos pueden ser utilizados, posteriormente, como herramientas y bases para otro tipo de estudios o para analizar la viabilidad de las obras en general. . Dos de los componentes principales del ciclo hidrológico son las precipitaciones y los flujos de los cuerpos de agua. De esta manera, la presente investigación realizó un análisis sobre el código estación pluviométrica (146008) y código estación fluviométrica (32620000), ambas ubicadas en el municipio de Cachoeira do Piriá, estado de Pará, con el objetivo de analizar la influencia de las precipitaciones. en los caudales del río en la cuenca hidrográfica mediante la construcción de un hietograma, realizando también la evaluación de la climatología normal y tendencia de la serie histórica de datos de lluvia, utilizando los métodos Lineal, Mann Kendall y Sen’s Slope. Para los datos de flujo se elaboró la curva de permanencia. Finalmente, se observó, a partir de los datos pluviométricos, que la precipitación en el municipio no presenta tendencia y, a partir de los datos fluviométricos, se pudo observar que su caudal en el $95 \%$ de los casos es, aproximadamente, $200 \mathrm{~m}^{3} / \mathrm{s}$.
\end{abstract}

Palabras clave: Precipitación; Tendencia; Caudal; Inundaciones.

\title{
1. Introdução
}

Desenvolver estudos que analisem o comportamento hidrológico em bacias hidrográficas é necessário, já que este pode ser utilizado posteriormente como ferramenta no desenvolvimento de obras hidráulicas, pois ajudam a evitar a ocorrência de alagamentos e consequentemente perdas econômicas (Correa \& Galvani, 2018). A precipitação é um importante componente do ciclo hidrológico e a partir da análise da mesma é possível verificar se a área de estudo apresenta períodos de sazonalidades de chuvas, obter o tempo de retorno para determinado volume precipitado, probabilidade de eventos extremos e entender como a chuva da região vem se alterando ao longo dos anos (Silva et al., 2019).

Segundo a Agencia Nacional de Águas (ANA, 2016) compreender o comportamento das chuvas de uma região ao longo dos anos é importante, já que este é um meio para percepção das mudanças no clima, haja vista que as mudanças climáticas acarretarão em possíveis alterações, seja no regime de precipitação, seja no escoamento dos corpos hídricos. De acordo com a World Resources Institute (WRI, 2020), as mudanças climáticas irão modificar os padrões de precipitação, alterando a intensidade e frequência das chuvas. O último relatório de avaliação do IPCC, o AR5, explica que o aumento gradual da temperatura, que presumidamente ocorrerá na maioria das regiões do planeta, é atribuído especialmente aos níveis de gases do efeito estufa (Schardong et al., 2014).

Uma maneira de constatar essas mudanças climáticas é através da análise de tendência em diferentes fatores climáticos, entre eles a precipitação (Alcântra et al., 2019). O estudo de tendência pode ser realizado por meio de diversos métodos, entre os mais conhecidos estão o de Mann Kendal, Spearman \& Linear, complementados pelo estimador Sen’s Slope. O teste de tendência não paramétrico de Mann Kendal é um dos mais utilizados na literatura (Sanche, Verdum \& Fisch 2013), sendo amplamente recomendado, até mesmo pela Organização Meteorológica Mundial (OMM).

Outra importante análise para o entendimento do ciclo hidrológico de uma região e suas possíveis alterações é a curva de permanência de seus rios. Compreender o comportamento da vazão dos rios e a influência das chuvas em seu volume disponível é algo necessário, para que os usos múltiplos não venham a prejudicar o volume disponível. A determinação da curva de permanência é uma ferramenta muito difundida entre os hidrólogos, mesmo não sendo a única metodologia disponível para determinar vazão outorgável, é certamente a mais utilizada por técnicos das secretarias de meio ambiente (Detzel, Fernandes \& Mine, 2016), considerando sempre a realidade de cada rio.

A “Amazônia Brasileira” é constituída por nove estados, setes deles são pertencentes a região norte, sendo eles: Acre, Amapá, Amazonas, Pará, Rondônia, Roraima e Tocantins, um na região nordeste, Maranhão, e um na região centro-oeste Mato Grosso, (Figueroa ,1990, Apud Silva et al, 2010). O município de Cachoeira do Piriá, está localizado no Estado do Pará, na bacia hidrográfica do rio Gurupi, pertencendo assim a Amazônia Brasileira, desta forma todos os estudos que possam ser relacionados e que apresentam relevância na área ambiental, não só nesse, mas em todos os municípios dentro dos nove estados componentes, 
são considerados relevantes. A avaliação de clima e precipitação na região é de crucial importância, como explica Borma et al, 2013 (Apud Lindemberg \& Silva, 2016):

"Eventos extremos de chuva alteram significativamente a vazão dos rios e são frequentemente muito prejudiciais para o ritmo de vida da população amazônica” (Borma et al, 2013, apud Lindemberg \& Silva, 2016, p.3).

Desta forma, fora realizado um estudo quantitativo, explicativo de cunho experimental, em concordância com a classificação apresentada por Estrela (2018). O objetivo do presente estudo foi realizar uma análise de tendência das chuvas ao longo dos anos, assim como o comportamento das vazões do rio principal sob a influência das precipitações da região e sua curva de permanência na região da bacia hidrográfica do rio Gurupi localizado no município Cachoeira do Piriá, PA, com a finalidade de verificar o comportamento hidrológico da região ao longo dos anos.

\section{Metodologia}

\subsection{Area de Estudo}

A área de estudo corresponde a bacia hidrográfica do rio Gurupi que está localizada na divisão de quatro municípios Cachoeira do Piriá e Viseu, ambos pertencentes ao estado do Pará e Boa Vista do Gurupi e Junco pertencentes ao estado do Maranhão, conforme o apresentado na Figura 1. A bacia hidrográfica do rio Gurupi corresponde a delimitação realizada e disponibilizada pela Agencia Nacional de Água com nível 6 de Otton codificação. A bacia hidrográfica apresenta uma área de drenagem de $451,85 \mathrm{~km}^{2}$.

Figura 1- Mapa de localização das estações Fluviométrica e Pluviométrica no Município de Cachoeira do Piriá, PA.

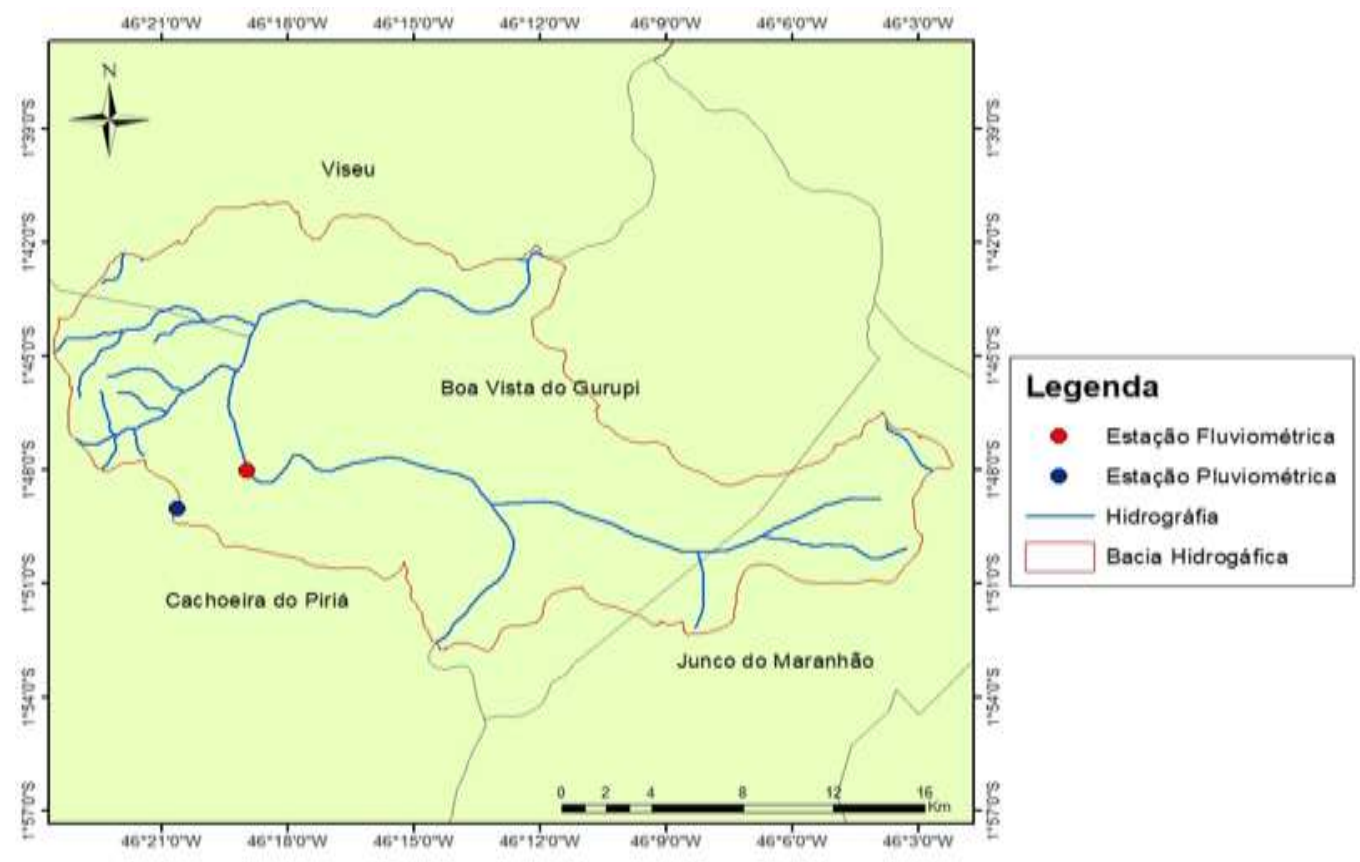

Fonte: Autores (2019).

As estações pluviométricas e fluviométricas estão localizadas no município de Cachoeira do Piriá que fica no estado do Pará. Segundo dados do Instituto Brasileiro de Geografia e Estatística (IBGE), a população do município de Cachoeira do Piriá, para o ano de 2010 era de cerca de 26.484 pessoas e densidade demográfica de $10,76 \mathrm{hab} / \mathrm{km}^{2}$, tendo uma estimativa populacional para o ano de 2018 de 33.178 pessoas. Ainda segundo o IBGE o Produto Interno Bruto (PIB) per capita do município era de cerca de $\mathrm{R} \$ 5.338,15$, além de possuir uma área territorial de cerca de $2.419,6 \mathrm{~km}^{2}$. 
Quanto a classificação de solo da bacia hidrográfica do rio Gurupi de acordo com o banco de dados do IBGE apresentado na Figura 2, observa-se a predominância de solo do tipo plintosolo e argissolo. O plintosolo é típico de zonas quentes e úmidas e o argissolo ocorre em diferentes condições climáticas (Embrapa, 2020).

Figura 2- Classificação de solos da bacia hidrográfica do rio Gurupi.

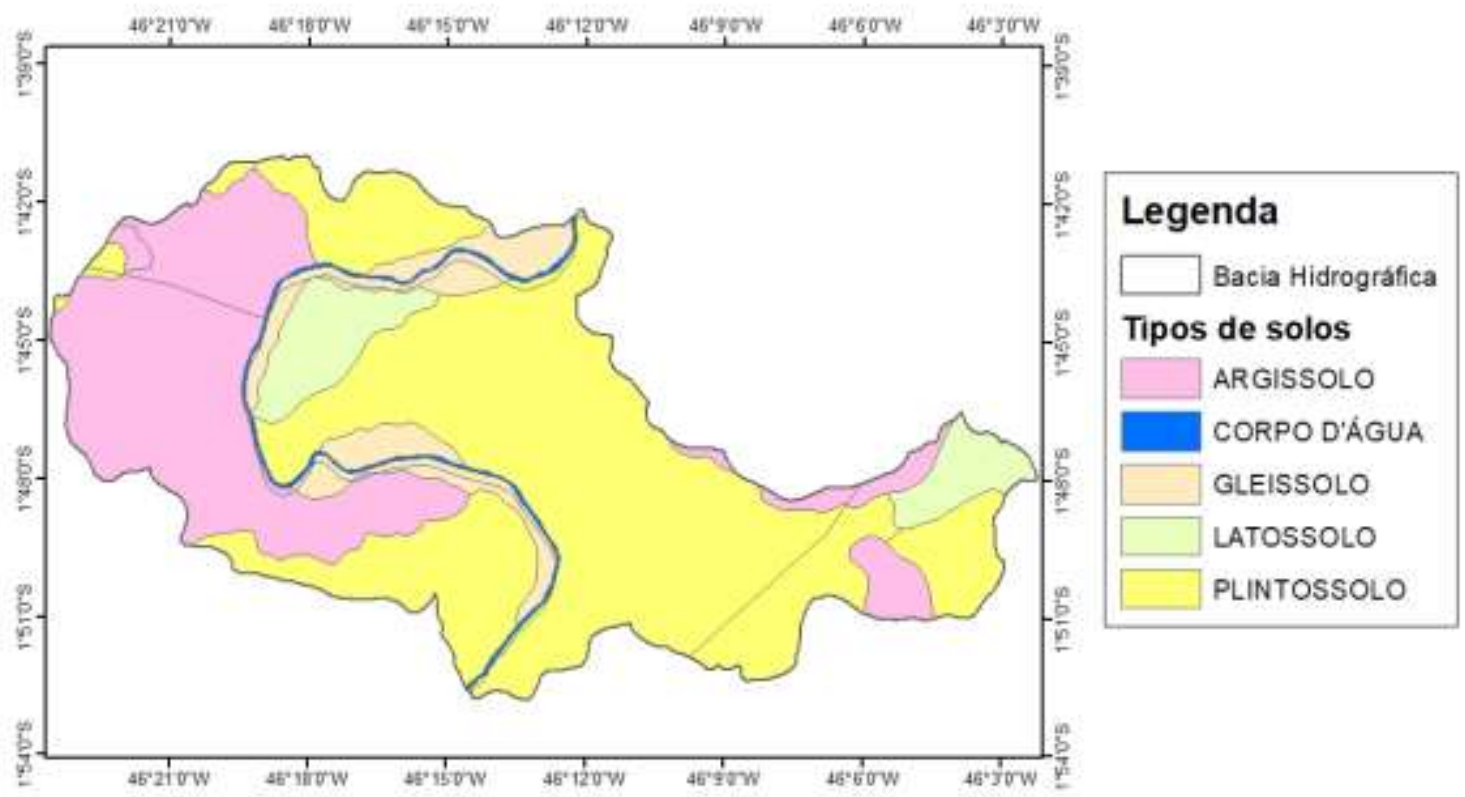

Fonte: Autores (2020).

No município de Cachoeira do Piriá predomina a vegetação rasteira, com umidade relativa do ar variando de 85 a $95 \%$. O clima predominante é equatorial úmido apresentando duas estações: inverno (período das chuvas de dezembro a maio) e verão (junho a novembro) (Lima, 2005). Na região do nordeste paraense onde se localiza Cachoeira do Piriá a precipitação pluviométrica fica, geralmente, no intervalo de 2.250 a $2.500 \mathrm{~mm}$ anuais (Silva \& Santos, 2006).

A metodologia utilizada na pesquisa é considerada de caráter quantitativo, seguindo o que é explanado por Estrela (2018), gerando assim uma resposta numérica. A aquisição dos dados e análise dos mesmos segue o molde de estudo experimental, ou seja, de cunho laboratorial, assim como na pesquisa de Progênio et al. (2019) que realizaram a análise de dados pluviométricos para a cidade de Belém do Pará. Pereita et al. (2018) explicam que os métodos quantitativos geram conjuntos ou massas de dados que podem ser analisados por meio de técnicas matemáticas de porcentagens, estatísticas e probabilidades, métodos numéricos, métodos analíticos e geração de equações e/ou fórmulas matemáticas aplicáveis a algum processo.

A metodologia, apresentada na Figura 3, foi dividida em três fases principais, são elas: $1^{\circ}$ ) Obtenção de dados pluviométricos e fluviométricos, ambos obtidos no site hidroweb da Agencia Nacional de águas; $2^{\circ}$ ) Organização dos dados de chuva e vazão, separadamente; $3^{\circ}$ ) Aplicação dos métodos de tendência e obtenção da normal climatológica, precipitação anual média e precipitação média mensal nos dados de precipitação, obtenção da curva de permanência para os dados de vazão e obtenção dos hietogramas para os dados da vazão e precipitação para a série curta de 10 anos. 
Figura 3- Fluxograma das etapas de metodologia do estudo.

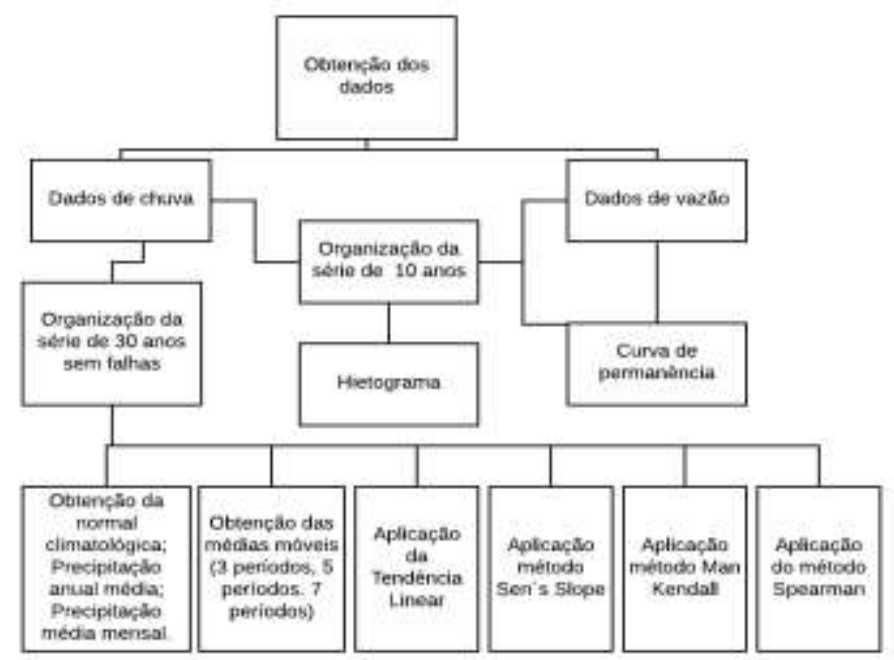

Fonte: Autores (2019).

Tratando especificamente da organização dos dados, os dados pluviométricos foram organizados do ano de 1985 a 2018, totalizando 34 anos, entretanto os anos de 1990, 2008, 2012 e 2013 apresentaram inconsistências e falhas, o que acarretou em suas respectivas retiradas da série, assim totalizando 30 anos de dados, sendo esse o número de anos mínimo recomendados pela Organização Mundial de Meteorologia (WMO). Já para os dados de vazão, foram organizados os dados dos últimos 10 anos, sendo eles de 2009 a 2018, entretanto para a efetiva correlação com os dados pluviométricos, foram também organizados os dados fluviométricos dos anos de 2006 e 2007, para que, ambas as séries estejam em períodos iguais. É importante ressaltar que foram utilizados dados de chuva de níveis de consistência 1 e 2, já que, caso algum nível fosse desconsiderado, haveria mais falhas na série, impossibilitando o estudo.

A partir dos dados já organizados de chuva, foram organizadas as precipitações mensais de cada um dos 30 anos, foram calculadas as precipitações médias para cada um dos dozes meses componentes do ano, após isso, a série foi dividia em 3 períodos, cada período composto por 10 anos de dados conforme mostra a Tabela 1. Após essa organização e cálculo das respectivas médias mensais para cada período, foi possível obter as normais climatológicas.

Tabela 1- Anos referentes a cada período.

\begin{tabular}{c|c|c}
\hline $1^{0}$ período & $2^{\text {}}$ período & $3^{\text {o }}$ período \\
\hline 1985 & 1996 & 2006 \\
\hline 1986 & 1997 & 2007 \\
\hline 1987 & 1998 & 2009 \\
\hline 1988 & 199 & 2010 \\
\hline 1989 & 2000 & 2011 \\
\hline 1991 & 2001 & 2014 \\
\hline 1992 & 2002 & 2015 \\
\hline 1993 & 2003 & 2016 \\
\hline 1994 & 2004 & 2017 \\
\hline 1995 & 2005 & 2018 \\
\hline
\end{tabular}

Fonte: Autores (2020). 
Foi aplicado o método de média móvel para analisar os dados de precipitação do município de Cachoeira do Piriá, para a série de dados de 30 anos, onde foram analisados 3 diferentes intervalos, o primeiro para 3 anos, o segundo para 5 anos e o ultimo para 7 anos. Com os resultados das médias móveis é possível analisar se há tendência nos dados de chuva e como a curva se comporta em diferentes intervalos.

Para a avaliação de tendência com os dados de chuva, foram aplicados alguns métodos, são eles: Tendência Linear, San's Slope, Man Kendall e Spearman. Para o método de tendência linear, os anos foram organizados de forma crescente, com seus respectivos dados pluviométricos, sendo posteriormente realizada a multiplicação entre a precipitação e seu respectivo ano de ocorrência (PxT), após a multiplicação, cada ano foi elevado ao quadrado $\left(\mathrm{T}^{2}\right)$, por fim, foi calculada o somatório dos anos, somatório das precipitações, somatório de PxT, e somatório de $\mathrm{T}^{2}$, objetivando a obtenção dos valores de alfa e beta da equação da reta (equação 1). Esse método objetiva estimar um valor condicional esperado, o que muitas vezes auxilia na simplificação da relação entre X e Y (Marcuzzo, Faria \& Filho, 2012)

$Y=\alpha+\beta X$

Eq. 1

Onde:

Y- Variável dependente Precipitação

$\alpha$ e $\beta$ - Coeficientes de regressão

$\mathrm{X}$ - Variável independente Ano

Para a aplicação do método de tendência Mann-Kendall (equação 2), foi realizado o teste estatístico que rejeita ou não a hipótese nula, como foi apresentado por Cabral Júnior \& Lucena (2020). Para que a hipótese nula seja rejeitada o módulo de Z deve ser maior que $Z \alpha / 2$. Considerando $\alpha$ de $5 \%$, o intervalo vai de $-1,96$ a $+1,96$, caso o valor de $Z$ encontrado estiver dentro do intervalo crítico, a tendência é considerada como não significativa.

$$
S=\sum_{I=2}^{N} \sum_{J=1}^{I=1} \operatorname{sinal}(X j-X i)
$$

Onde:

N- Número de anos da série

$\mathrm{Xj}$ - é o primeiro valor após $\mathrm{Xi}$

Para a aplicação do método de Spearman na série de dados de chuva, é necessário que os anos de precipitação sejam organizados em ordem crescente e enumerados $(1,2,3 \ldots)$ a esses nomeia-se Rxi, posteriormente as precipitações são organizadas em ordem crescente e enumeradas $(1,2,3 .$.$) e nomeia-se Ryi, assim é realizada a subtração entre Rxi e Ryi dos respectivos anos,$ eleva-se essa soma ao quadrado e assim obtêm-se os resultados para aplicação da equação 3, como relata Oscar Júnior (2019).

$R S=1-\left[\left(\frac{6}{n^{3}-n}\right) \sum_{i=1}^{n}(R x i-R y i)^{2}\right]$

Onde:

RS- Correlação de Spearman

N- Número de anos da série

Rxi- Valor ordenado de maneira crescente de anos

Ryi- Valor ordenado de maneira decrescente da precipitação 
Para que seja comprovada a existência de tendência na série de dados pluviométricos, a partir do método de Spearman, é necessário que o Tcal $\geq$ Tcr, "Caso o valor de tc seja superior ao valor crítico de t, devermos rejeitar a hipótese nula" Guimarães (2019), confirmando assim a existência de tendência.

Quanto aos dados de vazão, foram considerados dados diários dos últimos 10 anos, afim de obter o hietograma e a curva de permanência. Para a formulação do hietograma, resumidamente foram correlacionados dados de vazão e chuva para os mesmos períodos, como os dados de chuva apresentam falhas nos anos de 2008, 2012 e 2013, esses anos também foram retirados dos dados de vazão, assim os últimos 10 anos compreenderam de 2006 a 2018.

Para a formulação da curva de permanência, foi utilizada a metodologia da Eletrobras (Eletrobrás, 2000), o qual divide em grupos as vazões, sendo o intervalo entre esses grupos definido. Com os valores dos intervalos fechados, são contabilizados quantos dados se encontram dentro de cada grupo, assim é possível obter a porcentagem de cada grupo em relação ao total de dados da série. Assim, são organizadas as vazões em ordem decrescente e as porcentagens em ordem crescente, possibilitando a obtenção do gráfico referente a curva de permanência, por fim, ao observar o gráfico é possível obter o valor aproximado da vazão de ocorrência em $95 \%$ dos casos.

\section{Resultados e Discussão}

Foram analisados os dados de precipitação do município de Cachoeira do Piriá para um período de 30 anos que corresponde ao intervalo de 1885 a 2018 de série de dados. Segundo a Organização Mundial de Meteorologia, a normal climatológica é a média de dados climáticos de uma série de 30 anos. Na (Figura 4) estão apresentadas as normais climatológicas do município de Cachoeira do Piriá, foram realizadas médias mensais de precipitação para os 30 anos da série de dados, a partir da normal climatológica foi possível observar que a região apresenta características de sazonalidade de chuva. Nos meses de janeiro a junho os índices pluviométricos do município são maiores e nos meses de julho a dezembro o volume de chuva apresentado na região é menor, dados condizentes com dados relatados por Souza et al. (2016) para a Amazônia Brasileira, diferenciando apenas os meses de novembro e dezembro que para o município de Cachoeira do Piriá são mais secos e para um contexto geral da Amazônia Brasileira são meses com indicies pluviométricos expressivos.

Foram analisados também as normais climatológicas para três períodos de 10 anos dentro da série de dados de 30 anos. O $1^{\circ}$ período corresponde a 1985 a 1995 , exceto o ano de 1994 que não apresentou dados suficientes para ser analisado, o $2^{\circ}$ intervalo foi de 1996 a 2005 e o $3^{\circ}$ período foi de 2005 a 2018, exceto os anos de 2008, 2012 e 2013 não foram analisados. A análise que pode ser feita é que a média mensal apresentada pelo $1^{\circ}$ intervalo é que nos meses de janeiro, maio o volume precipitado foi maior que dos outros períodos. $\mathrm{O} 2^{\circ}$ período apresentou medias mensais maiores que os outros períodos apenas em 2 meses junho e setembro. $\mathrm{O} 3^{\circ}$ período apresenta índices de precipitação nos meses de fevereiro, março, abril, julho, agosto e dezembro maiores do que as médias mensais apresentadas nos intervalos anteriores. Mostrando que o volume precipitado nos anos mais recentes está aumentando, para a região. 
Figura 4- Normais Climatológicas.

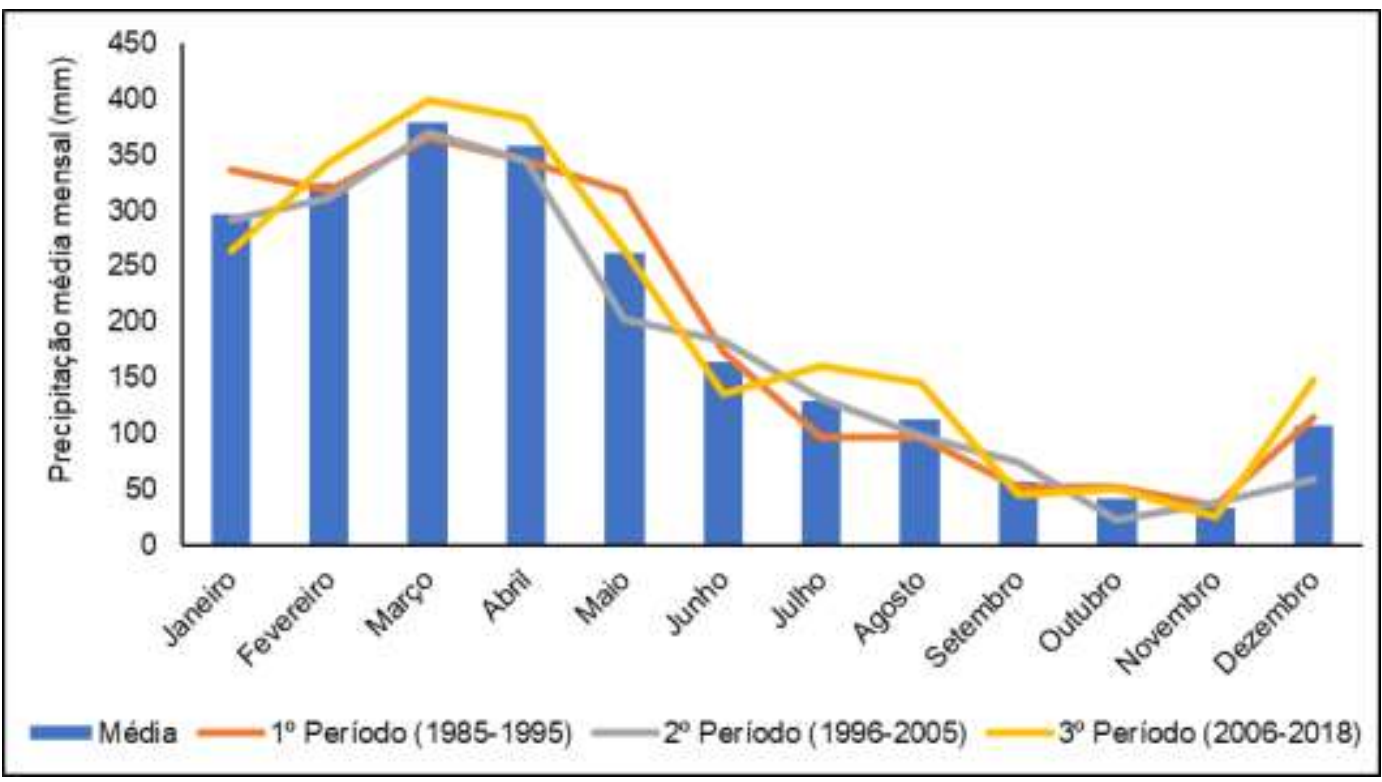

Fonte: Autores (2019).

Para a precipitação média anual dos 30 anos da série de dados, apresentado na (Figura 4) foi observado uma grande variabilidade entre as precipitações anuais, sendo o ano de 2018 o que apresentou maior média anual de precipitação $4.049,15$ mm, o ano de 1992 apresentou a menor média anual de chuva de 1.425,2 mm. Ainda na (Figura 4) estão apresentadas as médias móveis em relação a precipitação total anual dos 30 anos de dados, primeiramente foi realizada a média móvel para 3 anos, posteriormente foi feita a média móvel para 5 anos t e por fim foi realizada a média móvel para 7 anos, as médias móveis iniciam no gráfico a partir do ano central do intervalo. Foi possível analisar que quanto maior o intervalo em que a média móvel é feita, mas a curva apresentada pelos resultados será suavizada, como foi o caso do intervalo de 7 anos. Porém apenas com as médias móveis não foi possível determinar se o município de Cachoeira do Piriá apresenta tendência de chuva.

Figura 5- Precipitação média anual e médias móveis de Cachoeira do Piriá.

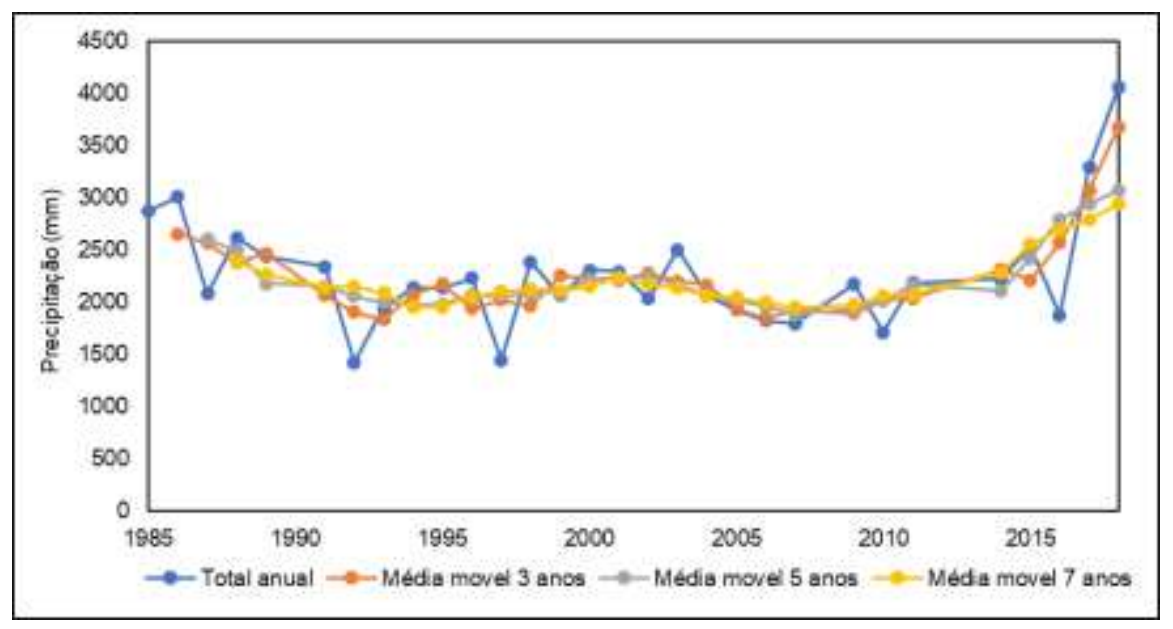

Fonte: Autores (2019). 
Dentre as precipitações mensais, foi possível obter a precipitação média mensal de 188,615 mm que está apresentada na (Figura 6) destacada em laranja, a maior precipitação mensal da série de dados dos 30 anos ocorreu em março de 2017 sendo está de $672 \mathrm{~mm}$, a segunda maior ocorreu em janeiro de 2000 e foi de 650,5 mm, alguns meses não apresentaram precipitação como o mês de novembro dos anos de 1987, 1991, 2009 e 2014, o mês de dezembro dos anos de 1991 e 2011, o mês de outubro dos anos de 1997 e 2006 e o mês de setembro do ano de 1997, todos esses meses se apresentam dentro dos meses com baixos índices pluviométricos, considerando a normal climatológica da apresentada na (Figura 4).

Figura 6- Precipitação mensal de Cachoeira do Piriá para 30 anos de dados.

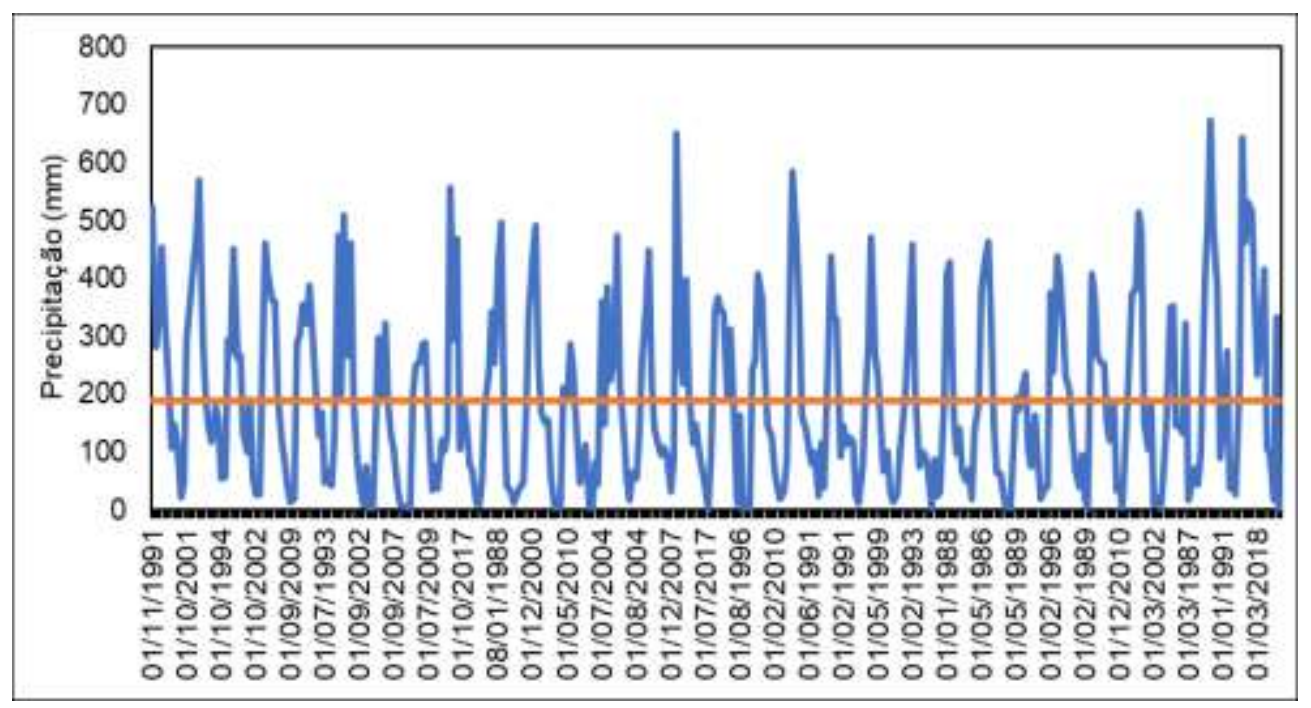

Fonte: Autores (2019).

Para identificar se o município de Cachoeira do Piriá apresenta tendência de crescimento e decrescimento nos seus índices anuais de precipitação foi aplicado o método de tendência linear para a série de 30 anos de dados. Foram calculados os valores de alfa e beta para obtenção da equação da reta, foram obtidos os valores de 7,16 para beta e -12056 para o valor de alfa, o gráfico apresentado na (Figura 7), mostra a série de dados anuais e a reta que representa a tendência linear. Conti (2000, apud Silva et al.,2018) afirma que a tendência é o aumento ou diminuição dos valores médio ao longo da série de dados, com no mínimo de 30 anos, que pode ou não ocorrer de forma linear, desta forma, é arriscada a aplicação somente do método de tendência linear, sem a comparação com outros métodos. 
Figura 7- Tendência linear de chuva a partir dos dados anuais de precipitação.

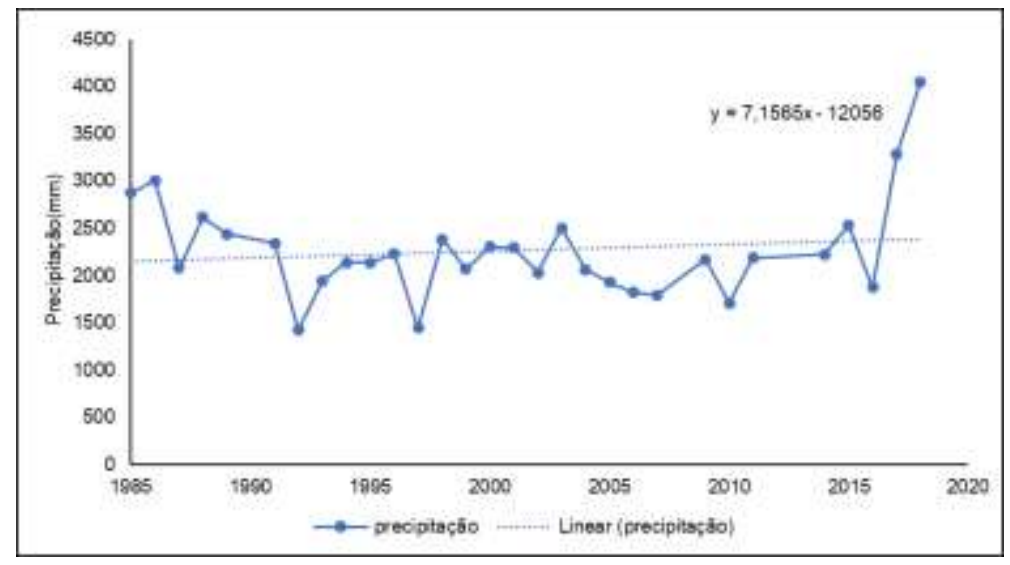

Fonte: Autores (2019).

O método de declive de Sen's Slope também foi aplicado a série de dados pluviométricos. O resultado da mediana foi de -7,03, esse método é utilizado para quantificar a inclinação e magnitude de uma tendência linear (Richard, 1987., Salmi, et al., 2002., Sen, 1968., Sneyers, 1975., apud SantoS et al, 2016). Moreira \& Naghettini (2016) explicam que valor positivo ou negativo da mediana indica tendência crescente ou decrescente para a série, no caso de Cachoeira do Piriá, segundo método de Sen's Slope, há indicação de tendência decrescente. Ao aplicar o método de Man-Kendall, considerando nível de significância de $5 \%$, temos que $Z \alpha / 2$ é 1,96, assim para ser considerado uma tendência significativa para o método de Man-Kendall é necessário que o valor de Z, deve ser <-1,96 ou >1,96, no caso de Cachoeira do Piriá, a aplicação do método resultou em um 0,607, ou seja, a hipótese nula não foi rejeitada e/ou não foi apresentada tendência significativa. O método de Spearman também foi aplicado a série de dados, onde o resultado apresentou um valor $\mathrm{T}$ calculado de aproximadamente $-0,47$ e $\mathrm{T}$ crítico de aproximadamente - 0,17 , o que mostra que o valor calculado é menor que o valor crítico, ou seja, a série não tem tendência.

Para a obtenção dos hietogramas e hidrogramas, foi necessário realizar a separação dos dados diários a cada ano, já que a série de chuva não se apresentava completa, gerando assim 10 gráficos que podem ser observados na (Figura 8).

É possível observar que os picos de precipitação, praticamente não ocorrem nos mesmos dias que os picos de vazão, isso pode ser explicado pelo fato de que só há escoamento superficial quando o aquífero já foi reabastecido, como relatado por Maciel (2017), além dos outros fatores que envolvem o ciclo hidrológico como um todo.

A menor vazão de $61,867 \mathrm{~m}^{3} / \mathrm{s}$, foi observada duas vezes no mês de dezembro do ano de 2016 e uma vez no mês de novembro de 2016, ambos os meses são considerados dentro da estação seca. O maior valor de vazão foi observado de 2488,632 $\mathrm{m}^{3}$ /s, ocorrida só uma vez, no mês de maio do ano de 2009, sendo esse mês considerado um mês chuvoso. 
Research, Society and Development, v. 10, n. 6, e38210615850, 2021

(CC BY 4.0) | ISSN 2525-3409 | DOI: http://dx.doi.org/10.33448/rsd-v10i6.15850

Figura 8- Hietogramas e hidrogramas para série de dados de 10 anos de Cachoeira do Piriá.

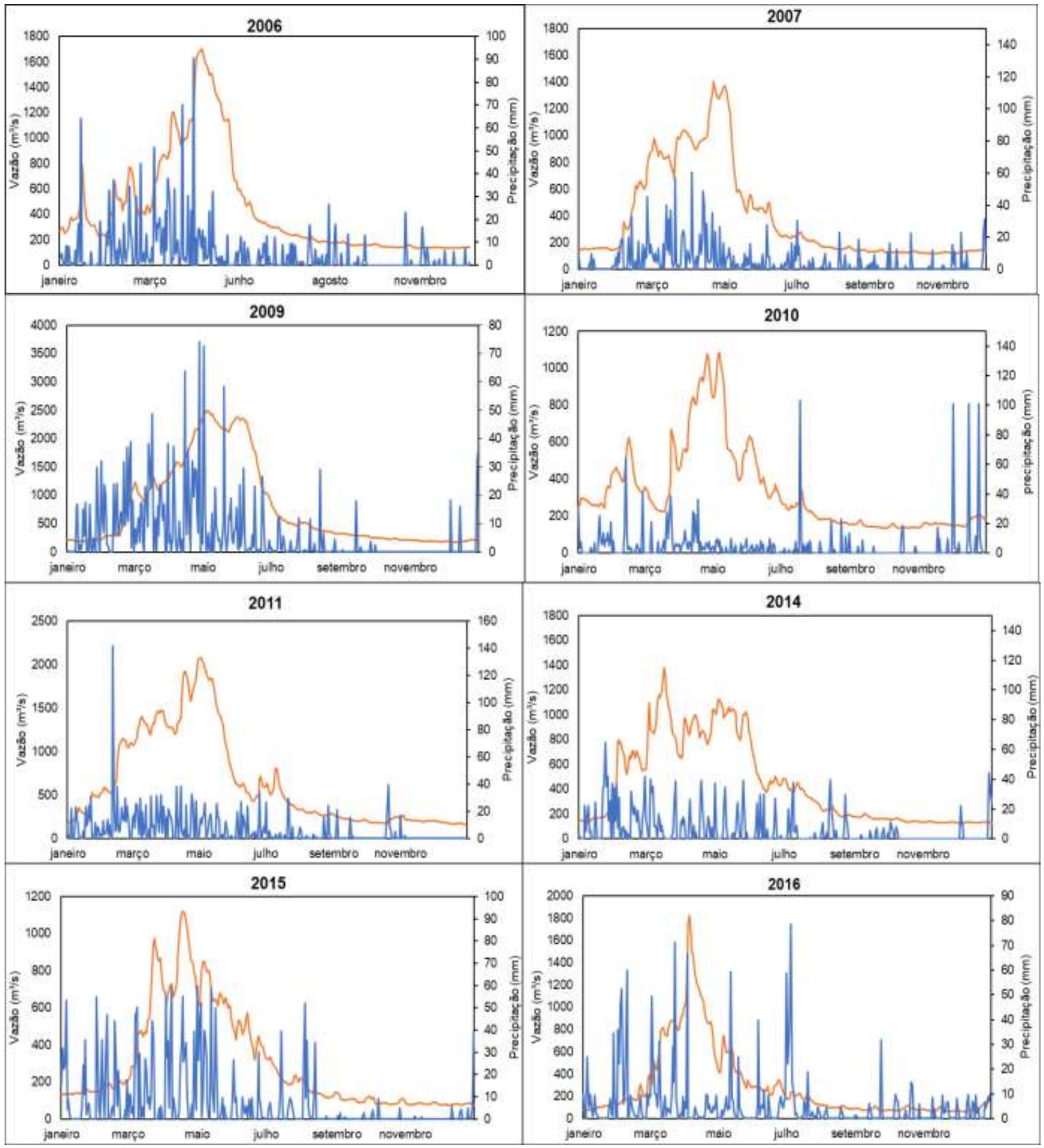




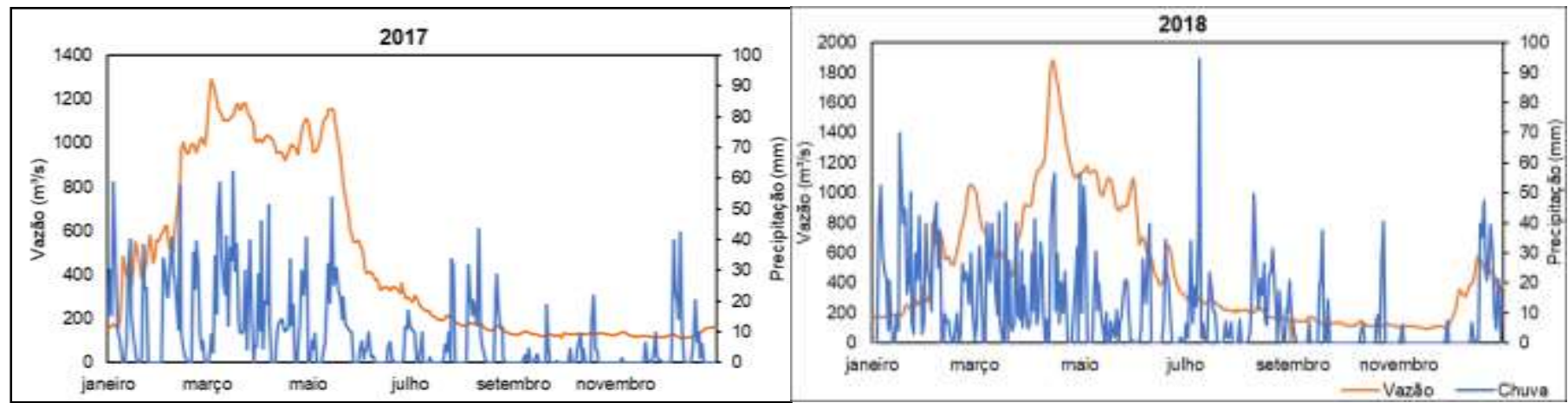

Fonte: Autores (2019).

Para a curva de permanência, a obtenção do valor da amplitude de cada intervalo resultou em 89,6501, resultando assim em 28 grupos. Assim, foram obtidas as porcentagens referentes a cada intervalo de vazão, sendo o intervalo variante entre $61,867 \mathrm{~m}^{3} / \mathrm{s}$ a $151,5178 \mathrm{~m}^{3} / \mathrm{s}$ o que apresenta $100 \%$ dos dados, assim foi possível obter o gráfico (Figura 9).

Seguindo o gráfico apresentado na figura 9, é possível observar que a vazão presente em 95\% da série, é de aproximadamente $200 \mathrm{~m}^{3} / \mathrm{s}$. Cruz e Tucci (2008, apud Marçal \& Silva, 2017) relatam a importância da curva de permanência como ferramenta para estabelecer a vazão de referência, para que seja mantido o equilíbrio do meio ambiente além de estabelecer os volumes das outorgas para os diversos usos. Cada órgão gestor do recurso hídrico, estabelece qual vazão de referência será utilizada, Q7,10, Q95\%, Q90\%, entre outras. De acordo com a Resolução CERH n ${ }^{\circ} 10$ de 2010 a vazão de permanência outorgada pela Secretaria de Meio Ambiente do Estado do Pará (SEMAS) Q95\%.

Figura 9- Curva de permanência do rio Gurupi.

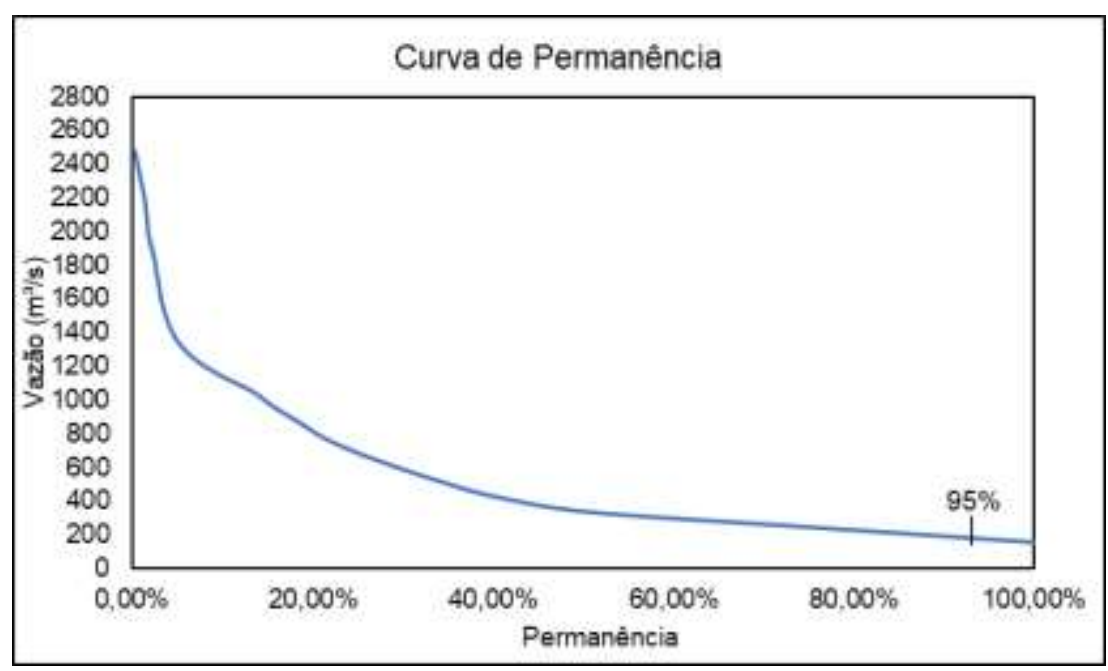

Fonte: Autores (2019).

\section{Conclusão}

Cachoeira do Piriá apresenta seus períodos secos e chuvosos bem distintos, onde o período chuvoso representa cerca de $72 \%$ do total anual, o período seco representa somente $17 \%$ da precipitação anual e os meses de transição (Dezembro e Junho) representam cerca de $12 \%$ das chuvas anuais, valores próximos ao encontrado por Coutinho et al. (2018) para toda a abacia Amazônica.

Quando comparados os métodos para identificação de tendência nas precipitações da região os resultados apresentaram uma pequena variação entre si. Os dados pluviométricos demonstram que, para o município de Cachoeira do Piriá, não há tendência nas precipitações, segundo os métodos de Man-Kendall e Spearman, entretanto, o método de Sen’s Slope mostrou que, 
segundo os dados de chuva, há uma diminuição de cerca de $-7,03 \mathrm{~mm}$ por ano na lâmina de chuva. Desta forma a diminuição que ocorre no município ainda não é considerada significativa.

Quanto a análise dos hietogramas, os dados de vazão apresentaram resultados esperados, onde, em meses chuvosos, ocorreram maiores vazões e em meses secos, ocorreram vazões menores. A partir da curva de permanência foi possível determinar a vazão de permanência Q95\% para a região de $200 \mathrm{~m}^{3} / \mathrm{s}$, podendo ser considerada uma vazão significativa. Entretanto é necessário o levantamento de mais dados fluviométricos para determinar a real vazão Q95\% para o rio Gurupi, que se estende por outros municípios.

É importante ressaltar a necessidade da realização de estudos mais detalhados para ambas as estações, fluviométricas e pluviométricas, além do preenchimento de falhas da série de dados de chuva, para que a correlação entre vazão e precipitação seja realizada de maneira mais minuciosa, resultando assim em valores mais próximos a realidade, dando embasamento mais forte aos estudos de cheias, inundações e outras variáveis afetadas pela precipitação no município, podendo assim auxiliar na resiliência da população passível de ser afetada e servindo como base de planejamento para os órgão públicos responsáveis.

Assim, sugere-se para o desenvolvimento de trabalhos futuros, que sejam analisadas outras estações fluviométricas ao longo do rio Gurupi, para que ocorra a determinação da curva de permanência para o rio como um todo, além da execução do preenchimento das falhas nos dados pluviométricos, o que possibilitará a realização de futuras análises pluviométricas continuas e de melhor representatividade.

\section{Referências}

Agência Nacional de Águas. (2016). Mudanças climáticas e recursos hídricos: avaliações e diretrizes para adaptação. ANA. https://www.ana.gov.br/todos-osdocumentos-do-portal/documentos-soe/mudancas-climaticas/mudanca-climatica-e-recursos-hidricos-2013-avaliacoes-e-diretrizes-para-adaptacao/mudancasclimaticas-e-recursos-hidricos-ana-2016.pdf

Alcântara, L. R. P. de et al. (2019). Análise de tendência para dados pluviométricos no município de Toritama-PE. Journal of Environmental Analysis and Progress, v. 4, n. 2, p. 130-139, http://www.ead.codai.ufrpe.br/index.php/JEAP/article/view/2377/482482963\#

Cabral Júnior, J. B., Lucena, R. L. (2020). Analysis of precipitation using mann-kendall and kruskal-wallis non-parametric tests. Mercator, 19, e19001. https://www.scielo.br/scielo.php?pid=S1984-22012020000100201\&script=sci_arttext\&tlng=pt

Correa, M. G. G. \&Galvani, E. (2019). correlação e variabilidade da precipitação e a vazão na bacia hidrográfica do rio piquiri - PR. Geo UERJ, 0(34), 40945. https://doi.org/10.12957/geouerj

Coutinho, E. de C. et al. (2018). Variabilidade climática da precipitação na bacia amazônica brasileira. Revista Brasileira de Climatologia, 22(0), 476-500. http://dx.doi.org/10.5380/abclima.v22i0

Detzel, Daniel Henrique Marco, Fernandes, Cristóvão Vicente Scapulatempo \& Mine, Miriam Rita Moro. (2016). Não Estacionariedade na Construção de Curvas de Permanência com Vistas à Outorga de Recursos Hídricos. RBRH, 21(1), 80-87. https://www.scielo.br/scielo.php?pid=S231803312016000100080\&script=sci_arttext\&tlng=pt

Empresa Brasileira De Pesquisa Agropecuária. (2000). Manual de pequenas Centrais Hidrelétricas. Ministério das Minas e Energia. https://www.embrapa.br/solos/sibcs/solos-do-brasil.

Estrela, C. (2018). Metodologia científica: ciência, ensino, pesquisa. (3ºd.). Porto Alegre: Artes Médicas. pp. $109-193$.

Guimarães, P. R. B. (2019). Análise de Correlação e medidas de associação. [capítulo não publicado]. Universidade Federal do Paraná. https://docs. ufpr. br/ jomarc/correlacao. pdf.

Instituto Brasileiro de Geografia e Estatística. (2010). Censo Demográfico. IBGE. https://censo2010.ibge.gov.br/

Lima, A. P. S. (2005). Avaliação do impacto de uma atividade garimpeira no município de Cachoeira do Piriá, Estado do Pará. [Tese doutoral, Instituto de Pesquisas Energéticas e Nucleares]. Repositório Ipen. http://repositorio.ipen.br/bitstream/handle/123456789/11220/10455.pdf?sequence=1\&isAllowed=y

Limberger, L. \& Silva, M. E. S. (2016). Precipitação na bacia amazônica e sua associação à variabilidade da temperatura da superfície dos oceanos Pacífico e Atlântico: uma revisão. GEOUSP Espaço e Tempo (Online), 20(3), 657-675. http://www.revistas.usp.br/geousp/article/view/105393.

Maciel, S. A. et al. (2017). Análise da relação chuva-vazão na bacia hidrográfica do Rio Paranaíba, Brasil. [Dissertação de Mestrado, Universidade Federal de Uberlândia]. Repositório UFU. http://repositorio.ufu.br/bitstream/123456789/18164/1/AnaliseRelacaoChuva.pdf

Marcuzzo, F. F. N, Faria, T. G. \& Pinto Filho, R. de F. (2012). Chuvas no estado de Goiás: análise histórica e tendência futura. ACTA Geográfica, 6(12), 125137. <http://rigeo.cprm.gov.br/xmlui/bitstream/handle/doc/615/Art_Chuvas_Marcuzzo.pdf?sequence=1\&isAllowed=y> Acesso em: 02 de dezembro de 2020. 
Research, Society and Development, v. 10, n. 6, e38210615850, 2021 (CC BY 4.0) | ISSN 2525-3409 | DOI: http://dx.doi.org/10.33448/rsd-v10i6.15850

Ministério do Desenvolvimento Agrário. (2006). Diagnóstico e planejamento de desenvolvimento do território rural do nordeste paraense. Ministério do Desenvolvimento Agrário. http://sit.mda.gov.br/download/ptdrs/ptdrs_territorio061.pdf

Moreira, J. G. do V. \& Naghettini, M. (2016). Detecção de Tendências Monotônicas Temporais e Relação com Erros dos Tipos I e II: Estudo de Caso em Séries de Precipitações Diárias Máximas Anuais do Estado do Acre. Revista Brasileira de Meteorologia, 31(4), 394-402. http://www.scielo.br/pdf/rbmet/v31n4/01027786-rbmet-0102-778631231420140155.pdf

Oscar Júnior, A. C. (2019). Homogeneização De Dados Pluviométricos Diários: Uma Contribuição Metodológica. Geo UERJ, 0(34), 40957,15 abr. 2019. https://doi.org/10.12957/geouerj

Pereira A. S. et al. (2018). Metodologia da pesquisa científica. [free e-book]. Santa Maria/RS. Ed. UAB/NTE/UFSM.

Progênio, M. F. ; Silva, G. R. A. ; Silva, F. S. \& CORDEIRO, A. L. M. (2019) . Análise da precipitação pluviométrica da cidade de Belém/PA. In: VIII Simpósio Internacional de Climatologia, Belém-PA.

Resolução Conselho Estadual de Recursos Hídricos nº 10, de 23 de setembro de 2010.

Rossi, M. S. \& Thebaldi, M. S. (2017). vazões de referência do rio são miguel em arcos (MG). Revista Agrogeoambiental, 9(1), 77-86. http://dx.doi.org/10.18406/2316-1817v9n12017927

Santos, A. P. P. dos et al. (2016). Precipitação na Cidade de Salvador: Variabilidade Temporal e Classificação em Quantis. Revista Brasileira de Meteorologia, 31(4), 454-467. http://www.scielo.br/pdf/rbmet/v31n4/0102-7786-rbmet-0102-778631231420150048.pdf

Schardong, A., Simonovic, S. P. \& Garcia, J. I. (2014). O possível efeito de mudanças climáticas e suas incertezas sobre afluências em sistemas de recursos hídricos. REGA, 11(2), 53-65. https://abrh.s3.sa-east-1.amazonaws.com/Sumarios/177/02b4d979 8cce9c5e488ed2e7d32b372e_074 3288d32 e633b2 5703d 8a9 259f3762.pdf

Silva, D. R. X. et al. (2010). Hanseníase, condições sociais e desmatamento na Amazônia brasileira. Revista Panamericana de Salud Pública, 27, 268-275. https://www.scielosp.org/scielo.php?pid=S1020-49892010000400005\&script=sci_arttext\&tlng=es.

Silva, E. R. A. C. et al. (2018). Análise da tendência temporal da precipitação pluviométrica interanual e intra-anual no semiárido pernambucano. Revista Brasileira de Climatologia, 22(0), 76-98. https://revistas.ufpr.br/revistaabclima/article/view/53956/34784.

Silva, R. M. G et al. (2019). Análise da variação pluviométrica do município de Cajazeirinhas-PB a partir de séries históricas/Analysis of the pluviometric variation of the municipality of Cajazeirinhas-PB from historical series. Brazilian Journal of Development, 5(7), 8074-8081. https://www.brazilianjournals.com/index.php/BRJD/article/view/2186/2219

Souza, E. B. D. et al. (2016). Sazonalidade da precipitação sobre a amazônia legal brasileira: clima atual e projeções futuras usando o modelo regcm4 (seasonal precipitation over the brazilian legal amazon: climate current and future projections using regcm4 model). Revista Brasileira de Climatologia, 18(0), 293-306. https://revistas.ufpr.br/revistaabclima/article/view/43711/28725.

World Resources Institute. (2020). Domestic Water Use Grew 600\% Over the Past 50 Years. WRI. https://www.wri.org/blog/2020/02/growth-domestic-wateruse 